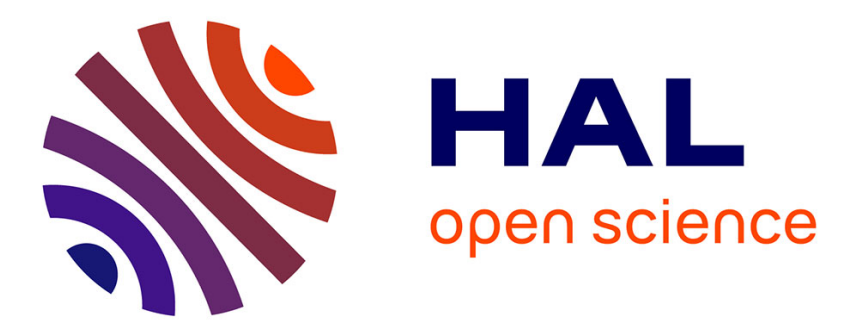

\title{
On the compromise between performance and robustness for viscoelastic damped structures
}

Kévin Jaboviste, Emeline Sadoulet, Nicolas Peyret, Charles Arnould, Eric Collard, Gael Chevallier

\section{To cite this version:}

Kévin Jaboviste, Emeline Sadoulet, Nicolas Peyret, Charles Arnould, Eric Collard, et al.. On the compromise between performance and robustness for viscoelastic damped structures. Mechanical Systems and Signal Processing, 2019, 119, pp.65-80. 10.1016/j.ymssp.2018.08.061 . hal-02370872

\section{HAL Id: hal-02370872 \\ https://hal.science/hal-02370872}

Submitted on 19 Nov 2019

HAL is a multi-disciplinary open access archive for the deposit and dissemination of scientific research documents, whether they are published or not. The documents may come from teaching and research institutions in France or abroad, or from public or private research centers.
L'archive ouverte pluridisciplinaire HAL, est destinée au dépôt et à la diffusion de documents scientifiques de niveau recherche, publiés ou non, émanant des établissements d'enseignement et de recherche français ou étrangers, des laboratoires publics ou privés.

\section{(c)(1)}

Distributed under a Creative Commons Attribution| 4.0 International License 


\title{
On the compromise between performance and robustness for viscoelastic damped structures
}

\author{
K. Jaboviste ${ }^{a}$, E. Sadoulet-Reboul ${ }^{a}$, N. Peyret ${ }^{c}$, C. Arnould $^{b}$, E. Collard $^{b}$, G. Chevallier ${ }^{a}$ \\ ${ }^{a}$ Univ. of Bourgogne Franche-Comté - FEMTO-ST Institute \\ Dpt of applied mechanics, 24, chemin de l'Epitaphe, 25000 Besancon, France \\ ${ }^{b}$ THALES LAS France, 2, avenue Gay Lussac, 78990, Elancourt, France \\ ${ }^{c}$ Laboratoire QUARTZ EA 7393 - 3 rue Fernand HAINAUT - 93400, France
}

\begin{abstract}
Viscoelastic materials are commonly used for passive treatment in many applications. They exhibit a specific behavior depending on frequency and temperature, and thus damping performances can strongly vary in an uncertain environment for which these materials can be used far from their optimal nominal value. The problem studied in this paper is the compromise to find between damping efficiency and robustness to lack-of-knowledge when using structure with viscoelastic components. This question is illustrated on a damper made of a steel frame, considering two kinds of viscoelastic materials : the tBA-PEGDMA which is a viscoelastic material that exhibits very good performances but a very sensitive behavior according to frequency and temperature, and the silicone rubber SI 965 which is less efficient but whose behavior is less frequency- and temperature-dependent than the tBA-PEGDMA. In order to evaluate the performances, a complex eigenvalue analysis is performed on the finite element model of the damper, with different rheological material models. In a very original way, the temperature is introduced in the model to investigate its influence on the modal damping. In this context, and using the developed methodologies, a robustness study is performed using the info-gap theory to evaluate the modal damping performances for the two considered viscoelastic materials in an uncertain temperature environment. It is shown that the best design choice in terms of viscoelastic behavior really depends on the degree of lack-of-knowledge : robust and better performances can be obtained while quantifying the horizon of uncertainty.
\end{abstract}

Keywords: Robustness, Info-Gap, Viscoelasticity, Damping, Vibrations

\footnotetext{
${ }^{1}$ E-mail adress: kevin.jaboviste@ @ femto-st.fr
} 


\section{Acronyms}

FEM : Finite Element Model

DOF : Degree Of Freedom

GMM : Generalized Maxwell Model

CEA : Complex Eigenvalue Analysis

FRF : Frequency Response Function

MAC : Modal Assurance Criterion

tBA-PEGDMA : tert Butyl Acrylate - Poly(Ethylen Glycol) Dimethacrylate

\section{Main symbols}

$K:$ stiffness matrix

$M$ : mass matrix

$K_{\text {elastic }}:$ stiffness matrix of the elastic part of the system

$K_{v i s c o}:$ stiffness matrix of the viscoelastic part of the system

$T_{g}$ : glass transition temperature

$U:$ displacement field 


\section{Introduction}

Viscoelastic materials are widely used for passive damping applications as for floor or dash panels [1] or for brake squeal mitigation [2] in automotive industry, in military aircraft and spacecraft industries [3]. They can be used as a free-layer or as a constrained layer treatment [4], as a multilayer laminate consisting of two skins with a viscoelastic core [5], or as a tuned viscoelastic mass damper [6]. Recent applications used such materials in assemblies to mitigate vibration transfers between parts inside structures, see [7]. When an excitation loading is applied to a viscoelastic material, dissipation occurs thanks to molecular rearrangements inside the material that lead to energy loss. These materials exhibit behaviors that depend on the temperature referred as the rubber phase and the glass phase separated by the glass transition. In the glass transition, the damping produced by the viscoelastic material is highest than in other temperature ranges. Moreover, viscoelastic materials are also known to depend on frequency or mechanical loadings for instance. Thus, if temperature fields and excitation loadings are badly known, it is difficult to optimize the geometrical design and to perform the material choice. In [7], a damping map, in a space of Young's modulus and loss factor, with characteristics evolution as a function of the temperature is plotted. It allows to travel in the design variable map and to optimize the targetted solution. One strategy can consist in using stable materials with low variations over the temperature range, but this can be at the expense of strong damping efficiency. It appears relevant to take into account the inevitable uncertainties in a design stage to ensure both damping performance and robustness. This design needs to be able to simulate the dynamic behavior of structures including viscoelastic components, and many difficulties arise from the modeling of the viscoelastic behavior, and from the multiple dependencies of this kind of material, see [8].

In this context, the work presented in this paper aims at comparing the performances in terms of damping and robustness of two chosen viscoelastic materials introduced in a damper made of a steel frame and polymer inclusions. Firstly, a method to model structures including viscoelastic elements with finite elements is proposed. This strategy is based on a state-space formulation, on a Generalized Maxwell Model and the projection of the mass and stiffness finite element operators on an adequate basis, [9, 2]. It allows to compute at reduced computation costs the complex eigenmodes and eigenfrequencies that are used to evaluate damping and modal performances. Secondly, the temperature dependency of different viscoelastic materials is highlighted and a very original way to build models to account for this dependency are proposed. Thirdly and finally, the damping efficiency of these materials is discussed in an uncertain context : the info-gap decision theory, which is well-suited for applications in which no probabilistic information is available, is used for that purpose. An application is performed on a damper made of a steel frame with viscoelastic inclusions.

\section{Finite element modeling of a viscoelastic damper}

This section aims at introducing both the use case and the theory basics of the simulation software especially developed for the application.

\subsection{Description of the proposed study case}

The study case consists in a damper made of a steel frame and viscoelastic elements added by melt-injection (Figure 1) and patented by THALES (WO2014111534A1). This damper is introduced in on optical image stabilizer fixed under fighter jets and aims to achieve a sufficient damping level in all the directions of space during application phases. It is mainly used to reduce transfers in the radial directions. In comparison with the mass of the entire damper (red part), the elastomer patches constitute a ratio of $2 \%$. The external ring is clamped on the optical devices while the internal ring is attached to the main frame. In this case, the vibration transmitted to the optics come from this structure. To accurately predict the physical behavior of the structure, this damper is modeled in details using the finite element method - FEM (Figure 1): the total mesh consists in 49527 quadratic tetrahedral elements.

The finite element simulation of structures including viscoelastic elements is not common due to the dependence of these materials to frequency or temperature especially. The use of a classical numerical model taking into account only the elastic behavior of the system allows to simplify the simulations but leads to inaccurate results. One way to represent the viscoelastic behavior is to introduce a complex temperature $(T)$-dependent and frequency $(\omega)$-dependent stiffness matrix $K^{*}(\omega, T)$ such that the dynamic discrete equations in the frequency domain can be written as,

$$
-\omega^{2}[M] \hat{U}(\omega, T)+\left[K^{*}(\omega, T)\right] \hat{U}(\omega, T)=\hat{F}
$$




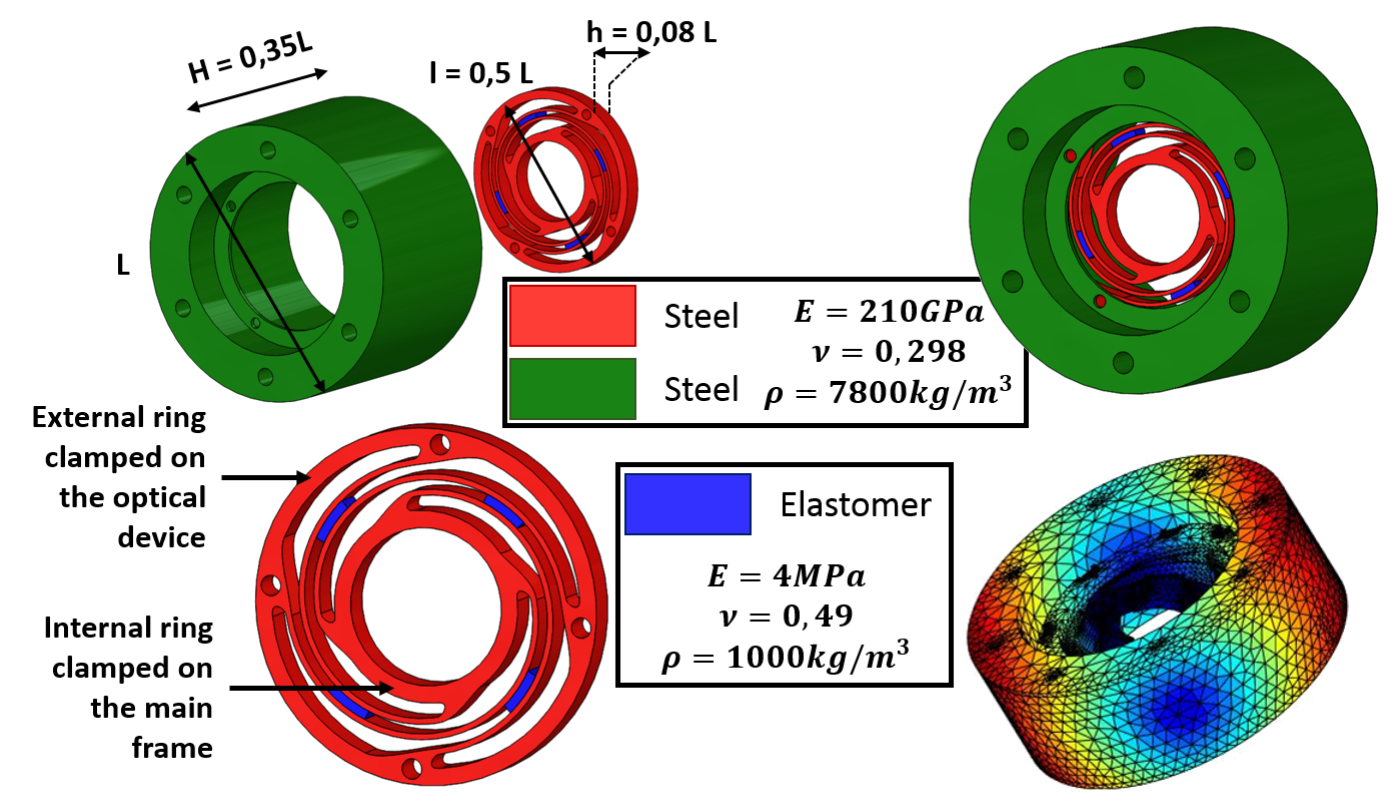

Fig. 1: Geometry materials, numerical model, mesh and studied mode shape

where $\omega$ is the frequency, $M$ is the real constant mass matrix, and $\hat{U}$ is the complex displacement vector. To represent linear viscoelastic materials, it is commonplace to use rheological models consisting of linear springs and dashpots connected in series or parallel. Many rheological model are available in the litterature $[10,11,12,13]$, the choice of an adequate model depends on the behavior exhibited by the material and on the time or frequency intended application domain.

\subsection{Rheological modeling of the viscoelastic elements}

The basic assumption of linear viscoelasticity is the existence of a relaxation function such that the stress is obtained as a convolution with the strain history. Using a Fourier transform, one obtains an equivalent representation where the material behavior depends on a complex modulus $E^{*}$ which depends on frequency $\omega$ and on temperature $T$. In many papers, it is divided into a real part, the storage modulus $E^{\prime}$, and an imaginary part, the loss modulus $E^{\prime \prime}$, such that,

$$
\hat{\sigma}(\omega)=E^{*}(\omega, T) \hat{\varepsilon}(\omega)=\left[E^{\prime}(\omega, T)+j E^{\prime \prime}(\omega, T)\right] \hat{\varepsilon}(\omega)
$$

The complexity of the rheological model depends on the behavior of the elastomer over the frequency and temperature range observed. Two elastomers are considered in the proposed study, and used as viscoelastic parts in the damper's finite element model. The first one is the tBA-PEGDMA [5] which is a shape memory polymer with changing behavior under thermal activation. The second one is a silicone rubber charged with black carbon particles. Figures 2 (a) and (b) present the storage modulus $E^{\prime}$ and the loss factor $\eta=\tan (\delta)=\frac{E^{\prime \prime}}{E^{\prime}}$ obtained using a dynamic mechanical analyzer for both polymers, as a function of the frequency for different temperatures in the case of the tBA-PEGDMA (a) where the time-temperature superposition principle is observed [14], and a function of temperature and for three frequencies $(1,10,100 \mathrm{~Hz})$ in the case of the silicone rubber $(\mathrm{b})$.

Both polymers exhibit typical viscoelastic behaviors : they change from a glass-like behavior at lowest temperature or highest frequencies, to a rubber-like behavior when the frequency decreases or the temperature is raised. The transition occurs at the glass-transition temperature and the materials then exhibit a very interesting loss factor at this specific temperature. For a studied temperature field variation included between $20^{\circ} \mathrm{C}$ and $80^{\circ} \mathrm{C}$ both materials 

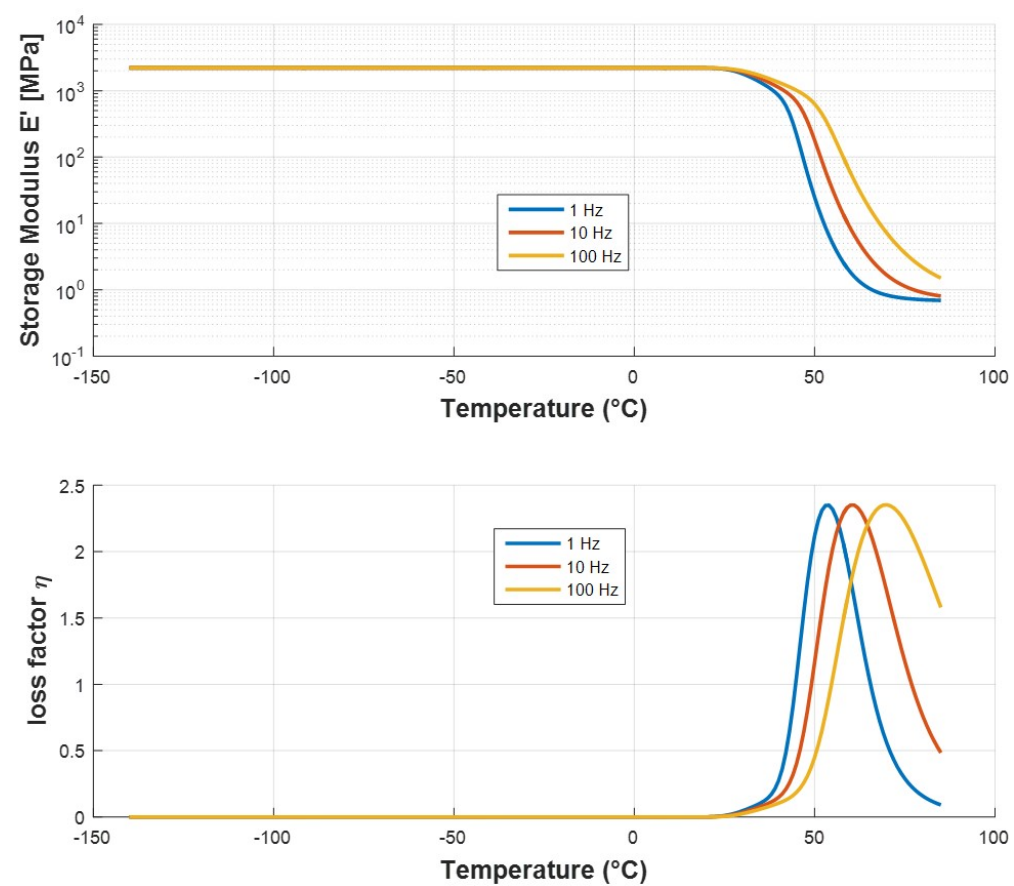

(a) tBA-PEGDMA: Storage modulus (top) and loss factor (bottom) according to the reduced frequency
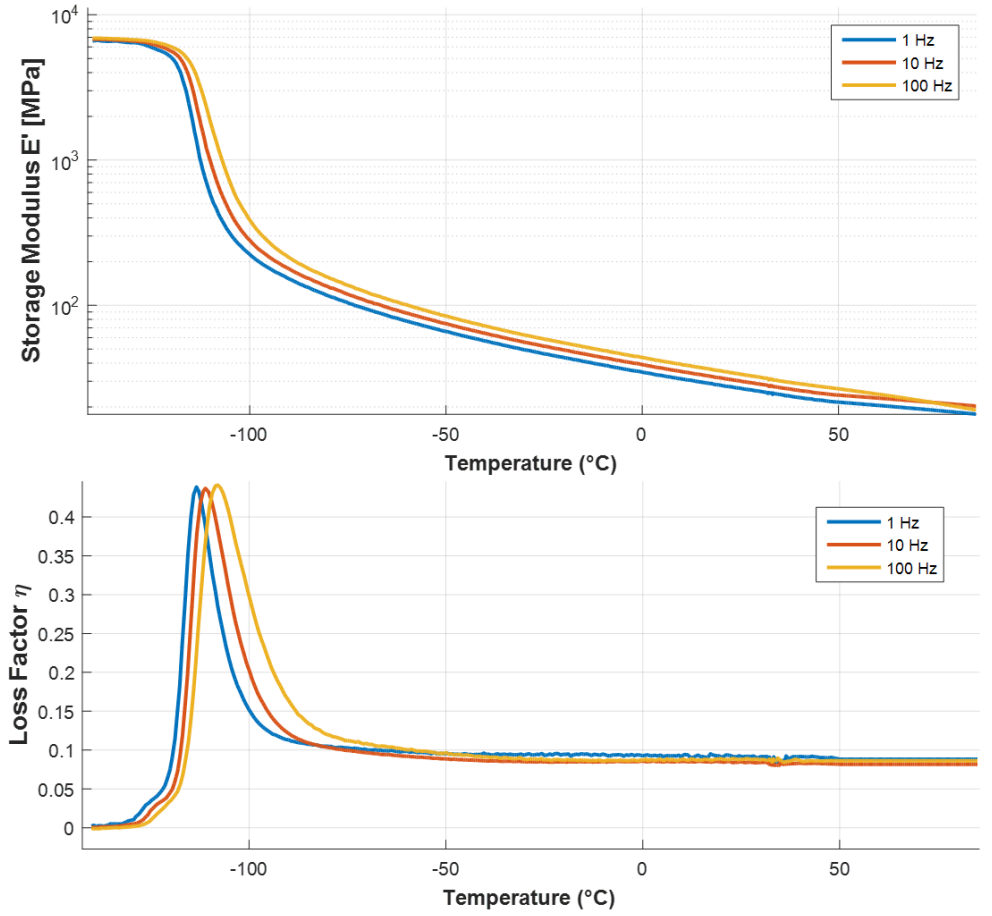

(b) Silicone rubber: Storage modulus (top) and loss factor (bottom) according to the temperature for three frequencies 1,10 and $100 \mathrm{~Hz}$

Figure 2: Viscoelastic properties of a tBA-PEGDMA shape memory polymer, and of a silicone rubber experimentally determined using a dynamic viscoelastic modulus analyzer 
present two different behaviors, and different rheological models have been chosen. A Generalized Maxwell Model $(\mathrm{GMM})$ is selected in order to describe all the evolution of the tBA-PEGDMA behavior. It consists in a series of linear Maxwell spring-dashpot cells in parallel with an equilibrium spring. This model can be adapted for studies on a large bandwidth, and thermal dependency can be integrated in the model parameters. The complex dynamic modulus can thus be written as follows,

$$
E^{*}=E_{\infty}+\sum_{i=1}^{n c e l l} \frac{E_{i} \tau_{i} j \omega}{1+\tau_{i} j \omega}=E_{\infty}\left(1+\sum_{i=1}^{n c e l l} \frac{\alpha_{i} \tau_{i} j \omega}{1+\tau_{i} j \omega}\right),
$$

where $E_{\infty}$ is the long term Young modulus, $E_{i}$ the dynamic modulus of the $i^{\text {th }}$ cell and $\tau_{i}$ its time constant. Increase the number of cells (ncell) allows the model to be consistent on a larger frequency range in return of an increase in the number of parameters. The second form is more adapted to the dynamic simulation. It introduces the frequency stiffening $\alpha_{i}$ as the ratio $E_{i} / E_{\infty}$. A structural damping is used to describe the silicone rubber behavior because the evolution of the storage modulus and the loss factor are almost linear in the range of temperature and frequency under study here. This rheological model is simpler than the GMM and the thermal dependency can be integrated in the model parameters too. The complex dynamic modulus can be written as follows,

$$
E^{*}=E_{s}(1+j \eta)
$$

where $E_{s}$ is the storage modulus and $\eta$ is the loss factor of the silicone rubber. These rheological models are then introduced in the dynamic finite element equations to solve (Eq. 1). The use of frequency and temperature dependent complex coefficients to represent the viscoelastic behavior leads to non-conventional problem to solve and a specific methodology is proposed here for a complex eigenvalue analysis.

\subsection{Complex eigenvalue analysis}

The homogeneous equation (Eq. 1) that describes the dynamic behavior of the damper with the rheological models respectively chosen for the silicone rubber and for tBA-PEGDMA and for an eigenvalue analysis can be written as follows,

$$
-\omega^{2}[M] \hat{U}+\left[K_{\text {elastic }}\right] \hat{U}+j \eta\left[K_{\text {visco }}\right] \hat{U}=0
$$

if the viscoelastic behavior is constant over the frequency range (structural damping) or as follows,

$$
-\omega^{2}[M] \hat{U}+\left[K_{\text {elastic }}\right] \hat{U}+\left[K_{\text {visco }}\right] \sum_{k=1}^{n \text { cell }} \frac{\alpha_{k} \tau_{k} j \omega}{1+\tau_{k} j \omega} \hat{U}=0
$$

if the viscoelastic behaviour is more complex (Generalized Maxwell Model). [ $\left.K_{\text {elastic }}\right]$ denotes the elastic stiffness matrix (i.e the addition of the stiffness matrices of the steel part and the silicone rubber patchs), $\left[K_{v i s c o}\right]$ is the stiffness matrix of the viscoelastic sub-domain completed with null terms to fit the size of the finite element model. ncell is the number of Maxwell cells in the GMM. $\alpha_{k}$ and $\tau_{k}$ are respectively the viscoelastic constant and the relaxation time for the $k^{\text {th }}$ cell of the model. To properly solve these equations, the system is transformed to get a state-space formulation of the problem. It is not useful with the structural damping model but it is unavoidable for the GMM in order to solve an generalized eigenvalue problem due to the frequency dependency of the parameters [2]. Introducing

$$
\hat{U}^{(d)}=j \omega \hat{U}
$$

the state formulation in the case of the structural damping model leads to:

$$
j \omega\left[\begin{array}{cc}
I & 0 \\
0 & M
\end{array}\right]\left[\begin{array}{c}
\hat{U} \\
\hat{U}^{(d)}
\end{array}\right]=\left[\begin{array}{cc}
0 & I \\
-K_{\text {elastic }}-j \eta K_{\text {visco }} & 0
\end{array}\right]\left[\begin{array}{c}
\hat{U} \\
\hat{U}^{(d)}
\end{array}\right]
$$

In the case of the Generalized Maxwell model, for one viscoelastic material in the mechanical device, an internal state is defined as,

$$
\hat{U}^{(k)}=\frac{\tau_{k}}{1+j \omega \tau_{k}} \hat{U}^{(d)}
$$


And the state formulation is written as follows:

$$
j \omega\left[\begin{array}{ccc}
I & 0 & 0 \\
0 & M & 0 \\
0 & 0 & B
\end{array}\right]\left[\begin{array}{c}
\hat{U} \\
\hat{U}^{(d)} \\
\hat{U}^{(k)}
\end{array}\right]=\left[\begin{array}{ccc}
0 & I & 0 \\
-K_{\text {elastic }} & 0 & -A \\
0 & d(B) & -I
\end{array}\right]\left[\begin{array}{c}
\hat{U} \\
\hat{U}^{(d)} \\
\hat{U}^{(k)}
\end{array}\right]
$$

Where :

$$
\begin{gathered}
B=\left[\begin{array}{ccc}
\tau_{1} I & 0 & 0 \\
0 & \ddots & 0 \\
0 & 0 & \tau_{\text {ncell }} I
\end{array}\right] \\
d(B)=\left[\begin{array}{c}
\tau_{1} I \\
\vdots \\
\tau_{\text {ncell }} I
\end{array}\right] \\
A=\left[\begin{array}{ccc}
\alpha_{1} K_{\text {visco }} & 0 & 0 \\
0 & \ddots & 0 \\
0 & 0 & \alpha_{\text {ncell }} K_{\text {visco }}
\end{array}\right]
\end{gathered}
$$

The main issue with the state-space formulation is the size of the system. For the structural damping model, the size is $2 * N_{D O F}$. For the GMM, it leads to $($ nbcell +2$) N_{D O F}$ where $N_{D O F}$ is the number of DOF in the initial finite element model. Moreover, the finite element models used for this kind of mechanical devices in the industrial domain are large-sized models which lead to crippling calculation times. Hence $N_{D O F}=249111$ is the case of the viscoelastic damper studied in this paper. In order to reduce the size, the mass $[M]$, the elastic stiffness $\left[K_{\text {elastic }}\right]$ and the elastomer stiffness $\left[K_{v i s c o}\right]$ matrices can be projected on a truncated basis. For the structural damping model associated with the silicone rubber, the weak evolution of the storage modulus and the loss factor according to the frequency and the temperature interval studied allows us to use a simple elastic eigenvectors basis $\left[\Phi_{e}\right]$. To obtain this basis, one can solve the following system to extract the $p$ first modes:

$$
\begin{aligned}
\left(-\omega_{p}^{2}[M]+\left[K_{\text {elastic }}\right]+\left[K_{\text {visco }}\right]\right) \phi_{p} & =0 \\
{\left[\Phi_{e}\right] } & =\left[\begin{array}{llll}
\phi_{1} & \phi_{2} & \ldots & \phi_{p}
\end{array}\right]
\end{aligned}
$$

The characteristics of the tBA-PEGDMA exhibit a stronger variation in the domain under study. To properly take into account the viscoelastic behavior and reduce the computation cost, the operators $[M],\left[K_{\text {elastic }}\right]$ and $\left[K_{\text {visco }}\right]$ are projected on a truncated multi-model basis $[15,16]$. In a dynamic problem with viscoelastic behavior, the multi-model approach allows us to build a projection basis that is representative of the viscoelastic material stiffening according to the rise of the frequency. One can choose three different frequencies to extract three truncated basis. The first frequency chosen is the minimum frequency of the studied interval. To create this "Low Frequency" (LF) basis $\left[\Phi_{L F}\right]$, the $p$ first modes are extracted by solving:

$$
\left(-\omega_{p}^{2}[M]+\left[K_{\text {elastic }}\right]+\operatorname{Re}\left(\left[K_{\text {visco }}\right] \sum_{k=1}^{n \text { cell }} \frac{\alpha_{k} \tau_{k} j \omega_{L F}}{1+\tau_{k} j \omega_{L F}}\right)\right) \phi_{p}=0
$$

The second frequency (MF) is localized around the glass transition temperature and the third frequency (HF) is the maximum frequency of the studied domain. The associated basis $\left[\Phi_{M F}\right]$ and $\left[\Phi_{H F}\right]$ are obtained solving the same equation used to extract $\left[\Phi_{L F}\right]$. Then, a modified Gram-Schmidt ortho-normalization is used to concatenate these three basis in one multi-model basis $[P]$.

In both cases, the projected operators will be noted : $[\mathrm{m}]$ for the mass, $\left[k_{\text {elastic }}\right]$ for the elastic stiffness and $\left[k_{\text {visco }}\right]$ for the elastomer stiffness.

Another way to reduce the computation cost is to assume that the relaxation times of the GMM can be selected "a priori" according to the frequency interval studied. One can take three relaxation times per decade. So it is assumed 

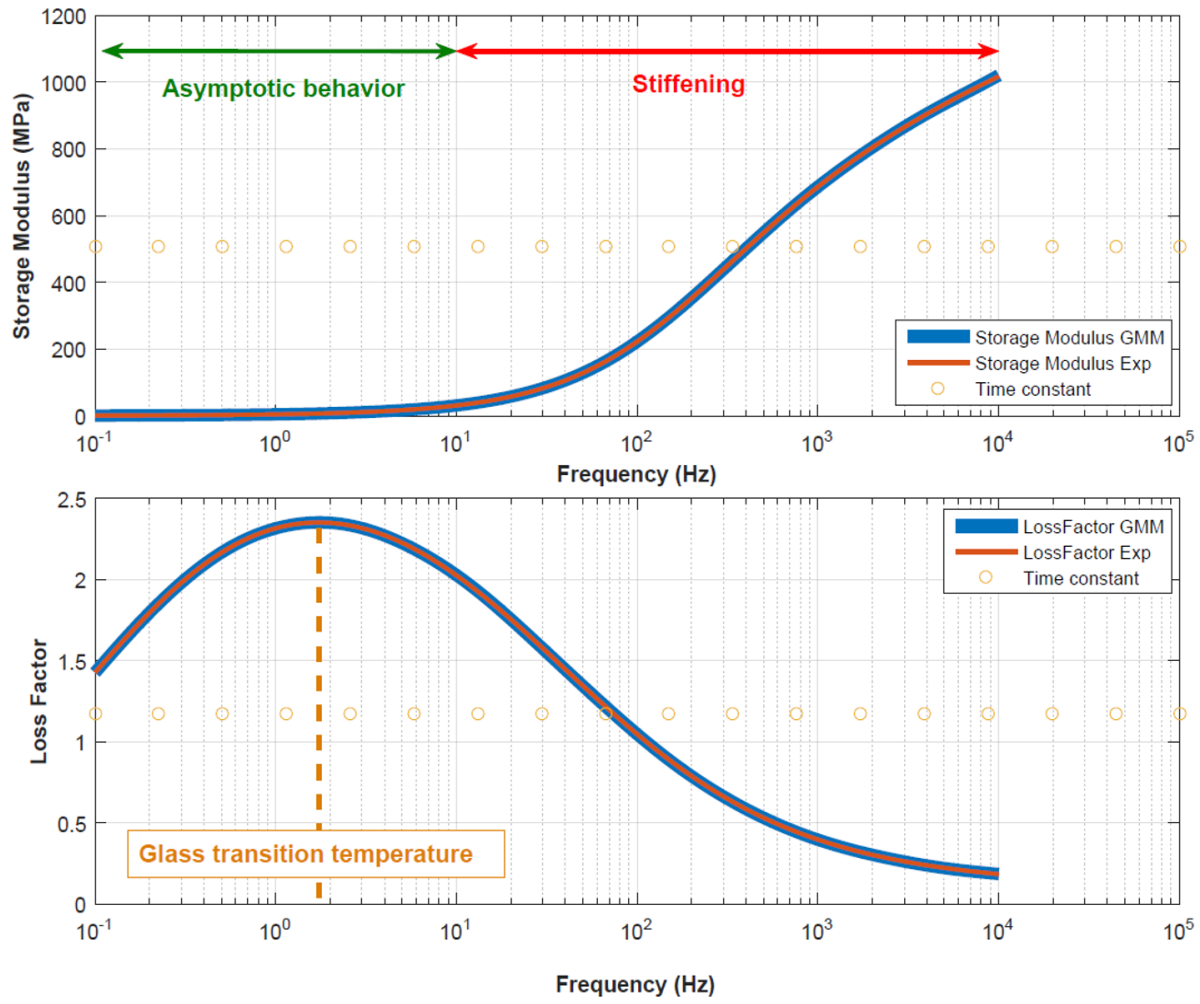

Fig. 3: Parameters identification of the GMM with chosen time constants

that these parameters no longer depend on the frequency. The identification of the GMM parameters under this assumption is still realistic [9] as shown Figure 3.

The state space formulation associated with the reduced order model and the assumption on the time constants for the GMM lead to a more affordable computation cost. The reduced state formulation for the structural damping model is:

$$
j \omega\left[\begin{array}{cc}
I & 0 \\
0 & m
\end{array}\right]\left[\begin{array}{c}
\hat{q} \\
q^{\hat{(d)}}
\end{array}\right]=\left[\begin{array}{cc}
0 & I \\
-k_{\text {elastic }}-j \eta k_{\text {visco }} & 0
\end{array}\right]\left[\begin{array}{c}
\hat{q} \\
q^{(d)}
\end{array}\right]
$$

where $q$ is the generalized coordinates and the reduced state formulation for the GMM model is:

$$
j \omega\left[\begin{array}{ccc}
I & 0 & 0 \\
0 & m & 0 \\
0 & 0 & b
\end{array}\right]\left[\begin{array}{c}
\hat{q} \\
q_{\hat{(d)}} \\
q^{\hat{(k)}}
\end{array}\right]=\left[\begin{array}{ccc}
0 & I & 0 \\
-k_{\text {elastic }} & 0 & -a \\
0 & d(b) & -I
\end{array}\right]\left[\begin{array}{c}
\hat{q} \\
q^{\hat{(d})} \\
q^{\hat{(k)}}
\end{array}\right]
$$

Where :

$$
\begin{gathered}
b=\left[\begin{array}{ccc}
\tau_{1} I & 0 & 0 \\
0 & \ddots & 0 \\
0 & 0 & \tau_{\text {ncell }} I
\end{array}\right] \\
d(b)=\left[\begin{array}{c}
\tau_{1} I \\
\vdots \\
\tau_{\text {ncell }} I
\end{array}\right]
\end{gathered}
$$




$$
a=\left[\begin{array}{ccc}
\alpha_{1} k_{\text {visco }} & 0 & 0 \\
0 & \ddots & 0 \\
0 & 0 & \alpha_{\text {ncell }} k_{\text {visco }}
\end{array}\right]
$$

Hence, the extraction of the complex eigenvalues $\omega_{i}$ and the complex eigenvectors $\phi_{i}$ is possible. This kind of simulation is called Complex Eigenvalue Analysis (CEA). In our case, the eigenfrequency and the modal damping associated are determined by:

$$
\begin{aligned}
f_{p i} & =\left|\omega_{i} / 2 \pi\right| \\
\zeta_{i} & =-\operatorname{Im}\left(f_{i}\right) / f_{p i}
\end{aligned}
$$

A validation of the method to take into account the viscoelastic behavior in the finite element model of the damper is needed. The french company THALES LAS France has made a lot of experimental tests on the proposed damper with the silicone rubber. These tests have allowed to identify the first resonance frequencies around a frequency ratio $R_{f}=0.3$ for the three rotations and around $R_{f}=0.57$ for the three translations. A sweep sine of $0.5 \mathrm{G}$ magnitude over the frequency range $[10 ; 1000] \mathrm{Hz}$ was used to excite the damper. The Complex Eigenvalue Analysis (CEA) is achieved and a diagram frequency-modal damping is plotted (Figure 4).

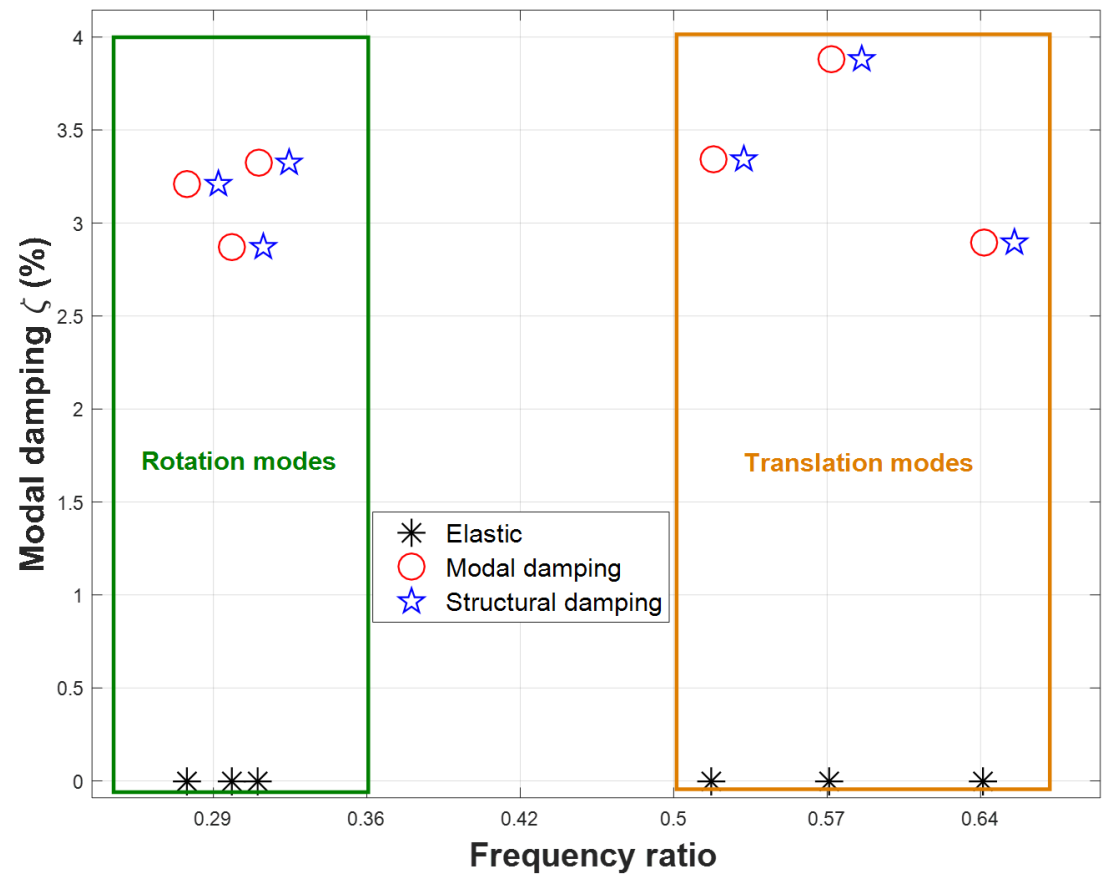

Fig. 4: Complex Eigenvalue Analysis: Elastic (black asterisk), Modal damping model (red circle) and structural damping model (blue star) system

A comparison between a viscoelastic system with modal damping values (red circle) and the same viscoelastic system with a structural damping model (blue star) is made. The CEA allows to take into account the dissipative behavior as well as the stiffness variation of the viscoelastic elements, and to compute the modal damping for each mode contribution. Here, the CEA is led with the structural damping model and one can notice a slight shift of the eigenfrequencies between the two viscoelastic problems while the identified frequencies of the elastic system (black asterisk) lie on the horizontal axis. Such a representation is useful to quantify the effects of the system variables (such as temperature) on the localization of the poles as showed further is the paper. In order to validate this analysis, the Frequency Response Function (FRF) of the full order model is calculated for a sweep sinus excitation on X direction. 
The frequency ratio and the associated modal damping $\left(R_{f}, \zeta\right)$ are compared for the $\mathrm{X}$ translation mode. From the FRF, the following values can be extract $R_{f}=0.525$ and $\zeta=0.034$ whereas, the CEA exhibits the following ones $R_{f}=0.528$ and $\zeta=0.035$. Thus, the CEA appears to correctly predict the modal behavior of the system.

\section{Effect of temperature on the dynamic behavior of a viscoelastic damper}

Due to the important temperature variation observed in the aeronautic domain, it is necessary to study the dynamic behavior of mechanical devices using viscoelastic materials according to this parameter. Hence, the aim of this section is to compare the modal damping of each viscoelastic damper on the first modes according to the temperature variation.

\subsection{Modeling of the viscoelastic behavior according to the temperature in the structural damping and generalized Maxwell models}

The damping behavior of viscoelastic materials strongly depends on several parameter such as temperature and numerous studies have highlighted this phenomenon and have proposed methods to deal with [17, 18, 19, 20, 21]. Despite the knowledge of the temperature dependency, it seems to be still difficult to take it into account in classical damping model such as structural damping, Zener or Generalized Maxwell model for example, when the timetemperature superposition principle is not observed. That is why it is proposed, for the studied temperature field (between $20^{\circ} \mathrm{C}$ and $80^{\circ} \mathrm{C}$ ), to use two different rheological models to describe the silicone rubber and tBA-PEGDMA behavior. It should be noticed that the loss factor of the silicone rubber is almost constant in our case (Figure 2b) and its storage modulus decrease linearly (Figure 5).

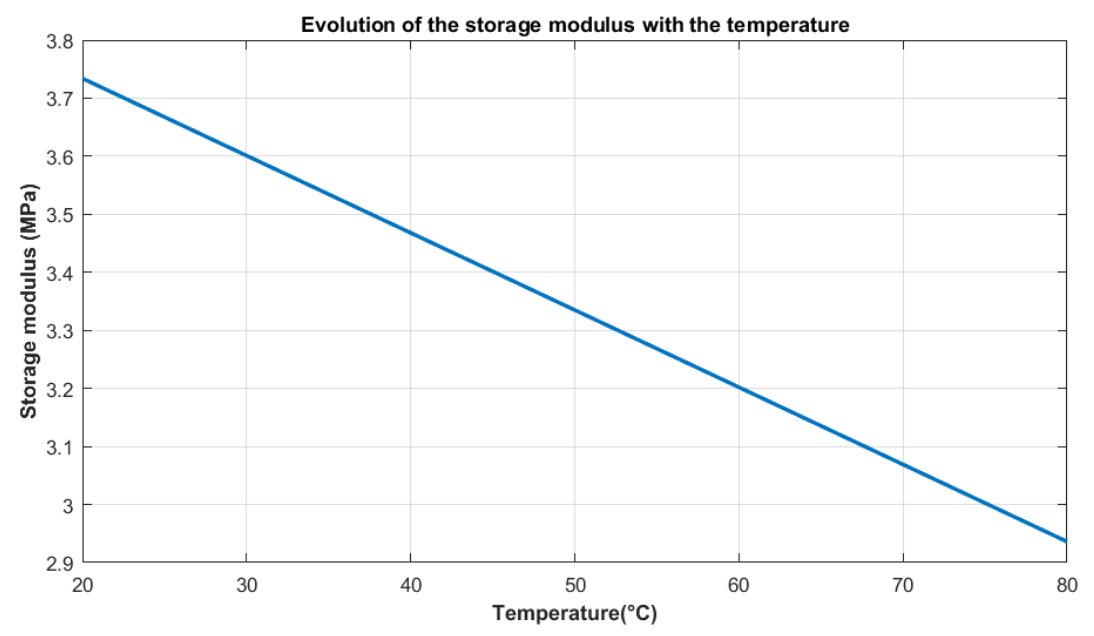

Fig. 5: Storage modulus evolution of the silicone rubber according to the temperature

Hence, the stiffness matrix of the viscoelastic part $\left[K_{v i s c o}\right]$ will vary linearly and the homogeneous equation of the damper with the silicone rubber parts can be written as follows :

$$
-\omega^{2}[M] \hat{U}+\left[K_{\text {elastic }}\right] \hat{U}+j \eta\left[K_{\text {visco }}(T)\right] \hat{U}=0
$$

The tBA-PEGDMA behavior is represented by a GMM where there is already a assumption on the time constants. These parameters do not depend on frequency any more. Now, it is assumed that they do not depend on temperature too. Considering the number of parameters, the identification problem is an under-constrained optimization problem thus the added constraint leads to a better definition of the optimization problem that ensures existence and uniqueness of the set of identified parameters. The evolution of the storage modulus and the $\alpha$ coefficients is complex. To properly extract this information, an identification of these parameters is leaded for each temperature step thanks to the Least Square method ("fmincon" Matlab function) to fit the experimental results. The initialization of the first loop is made 
by solving the following linear system at the minimum temperature of the studied domain:

$$
\left[\begin{array}{cccc}
1 & -\frac{1}{1+\tau_{1}^{2} \omega_{1}^{2}} & \cdots & -\frac{1}{1+\tau_{N}^{2} \omega_{1}^{2}} \\
0 & \frac{\tau_{1} \omega_{1}}{1+\tau_{1}^{2} \omega_{1}^{2}} & \cdots & \frac{\tau_{N} \omega_{1}}{1+\tau_{N}^{2} \omega_{1}^{2}} \\
1 & -\frac{1}{1+\tau_{1}^{2} \omega_{2}^{2}} & \cdots & -\frac{1}{1+\tau_{N}^{2} \omega_{2}^{2}} \\
0 & \frac{\tau_{1} \omega_{2}}{1+\tau_{1}^{2} \omega_{2}^{2}} & \cdots & \frac{\tau_{N} \omega_{2}}{1+\tau_{N}^{2} \omega_{2}^{2}} \\
\vdots & \vdots & \cdots & \vdots \\
1 & -\frac{1}{1+\tau_{1}^{2} \omega_{M}^{2}} & \cdots & -\frac{1}{1+\tau_{N}^{2} \omega_{M}^{2}} \\
0 & \frac{\tau_{1} \omega_{M}^{2}}{1+\tau_{1}^{2} \omega_{M}^{2}} & \cdots & \frac{\tau_{N} \omega_{M}^{2}}{1+\tau_{N}^{2} \omega_{M}^{2}}
\end{array}\right]\left[\begin{array}{c}
E_{0} \\
E_{1} \\
\vdots \\
E_{N}
\end{array}\right]=\left[\begin{array}{c}
E_{s}^{\omega_{1}} \\
E_{l}^{\omega_{1}} \\
\vdots \\
E_{s}^{\omega_{M}} \\
E_{l}^{\omega_{M}}
\end{array}\right]
$$

Where $\omega_{1}, \omega_{M}$ are, respectively, the pulsation associated to the minimum and the maximum frequency on the studied domain. $N$ is the number of the last cell used in the GMM. $E_{s}$ and $E_{l}$ are the experimental results and the sought parameters are the storage modulus $E_{0}$, and the dynamic stiffness $E_{1 \ldots N}$. This first initialization has to be performed on the asymptotic domain of the viscoelastic storage modulus. Then, the initial point of the next loop is the set of identified parameters coming from the previous step. The evolution of the long term modulus $E_{\infty}$, the dynamic stiffness $E_{k}$ and the dynamic stiffness ratios $\alpha_{k}$ according to the temperature for the GMM is shown Figure 6 . The temperature interval is larger than the one used before to observe the silicone rubber behavior. Here the temperatures are included between $10^{\circ} \mathrm{C}$ and $200^{\circ} \mathrm{C}$ to capture all the variation of the $\alpha$ coefficients. In fact, the tBA-PEGDMA switch from its glassy phase (before $20^{\circ} \mathrm{C}$ ) to its rubbery phase $\left(\right.$ after $160^{\circ} \mathrm{C}$ ) on this interval. Moreover, the dynamic stiffness $E_{k}(T)$ exhibit almost the same variation according to the temperature for each cell. For the cells 1,2 , and 3 associated to the lowest time constant and the cells 19,20, and 21 associated to the highest time constant, it seems that the variations are irregular due to border effects. The stiffness ratio $\alpha_{k}$ shows a more complex evolution according to the temperature. It can be explain by the following relation obtain from the equation (Eq. 3) for one cell $k$ when $\omega_{k}=1 / \tau_{k}: \alpha_{k}(T)=E\left(\omega_{k}, T\right) / E_{\infty}(T)-1$.

Hence, in the finite element model of the viscoelastic insulator, the stiffness matrix of the viscoelastic part [ $\left.K_{\text {visco }}\right]$ and the $\alpha$ coefficients depend on the temperature. The homogeneous equation of the damper with the tBA-PEGDMA parts can be written as follows:

$$
-\omega^{2}[M] \hat{U}+\left[K_{\text {elastic }}\right] \hat{U}+\left[K_{\text {visco }}(T)\right] \sum_{k=1}^{n c e l l} \frac{\alpha_{k}(T) \tau_{k} j \omega}{1+\tau_{k} j \omega} \hat{U}=0
$$

\subsection{Complex Eigenvalue Analysis according to the temperature}

Both the silicone rubber and tBA-PEGDMA temperature dependency are taken into account in their respective rheological models and "in fine" in the finite element model of the damper. With these models in temperature, one can drive CEA at several temperature and observed the behavior of the whole damper in terms of eigenfrequency and modal damping.

The Figure 7 shows the variation of the pole locations according to the temperature for the damper with the silicone rubber part. Only the first six poles are followed for a temperature included between $20^{\circ} \mathrm{C}$ and $80^{\circ} \mathrm{C}$.

The evolution of the poles is smooth as one would expect. Due to the softening of the silicone rubber stiffness according to the temperature, one can observe a drop of the eigenfrequencies of the system around $8.3 \%$. This loss of stiffness leads also to a reduction of the modal damping about $16.7 \%$.

As it is shown Figure 8, the variation of the pole locations according to the temperature for the damper with the tBA-PEGDMA parts is more complex than the evolution with the silicone rubber parts. Only the first four poles are plotted for a temperature included between $10^{\circ} \mathrm{C}$ and $200^{\circ} \mathrm{C}$ to capture all the behavior variations.

One can notice that the pole evolutions are not continuous and there are some jumps between their different paths according to the temperature. This kind of phenomenon can be explained if some changes occur in the vibration modes of the damper. So, if the aim is to compare the two elastomers and their impacts on the poles location, a tool is 

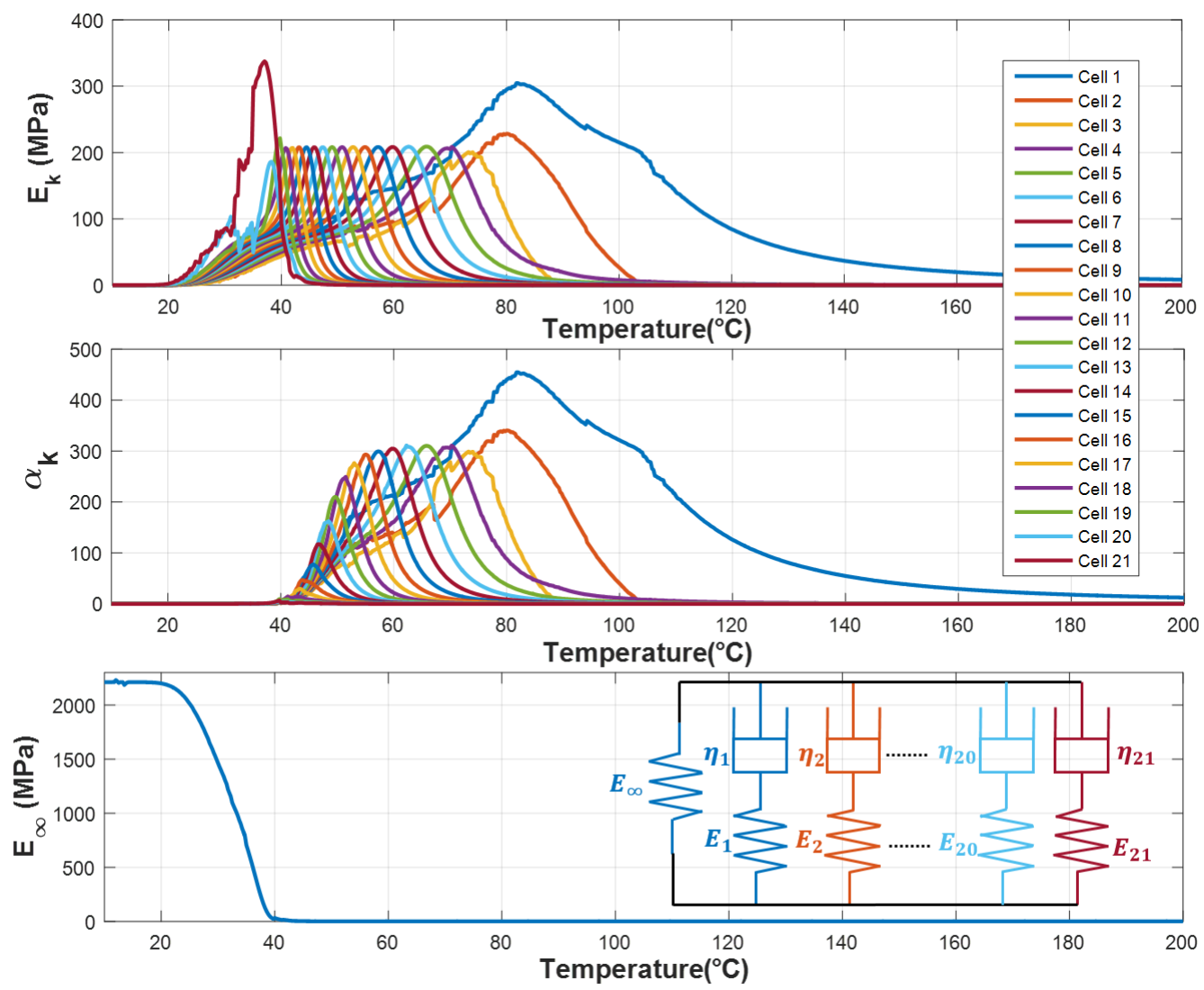

Fig. 6: Dynamic stiffness $E_{k}$, dynamic stiffness ratio $\alpha_{k}$ and long term modulus $E_{\infty}$ evolution of the tBA-PEGDMA according to the temperature

needed to track them over all the studied temperature interval. To properly achieve this goal, an index is created based on the Modal Assurance Criterion (MAC) principle. The mode $j$ to be tracked is extracted at $10^{\circ} C: \phi_{j}\left(10^{\circ} C\right)$. Then the collinearity between this mode and each vector of the truncated basis at each temperature step $[\Phi(T)]$ is computed. For each step, the index evaluates the number of the most collinear vector of the basis. The index expression at one temperature is given below:

$$
\operatorname{index}(T)=\frac{\left|\phi_{j}\left(10^{\circ} C\right)^{H} \cdot[M] \cdot[\Phi(T)]\right|^{2}}{\phi_{j}\left(10^{\circ} C\right)^{H} \cdot \phi_{j}\left(10^{\circ} C\right) \cdot[\Phi(T)]^{H} \cdot[\Phi(T)]}
$$

Where $H$ is the Hermitian transposition of the matrix and $T$ will vary from $10^{\circ} C$ to $200^{\circ} C$. Hence, the chosen mode $j$ is tracked all along the temperature range as shown Figure 9 a for the $4^{\text {th }}$ mode of the damper. It becomes possible to reconstruct this mode and plot its pole location according to the temperature (Figure 9b).

The evolution of the $4^{\text {th }}$ pole of the damper with the tBA-PEGDMA parts is important according to the temperature. This phenomenon comes from the larger temperature interval observed. In fact, the glass transition temperature of the tBA-PEGDMA is included in this interval. So, one can notice that the eigenfrequency decreases all along the temperature rise and moves from a frequency ratio $R_{f}=0.908$ to $R_{f}=0.085$. It comes from the important loss of the tBA stiffness at the glass transition temperature, from $2211 \mathrm{MPa}$ to $0.47 \mathrm{MPa}$. At the extremities of the temperature range, the modal damping of the $4^{\text {th }}$ mode in around $1.5 \%$ but the maximum is reached around $50^{\circ} \mathrm{C}$ with a modal damping near to 55\%. Finally, there is a last notable phenomenon around $R_{f}=0.6$. The jump observed on the 


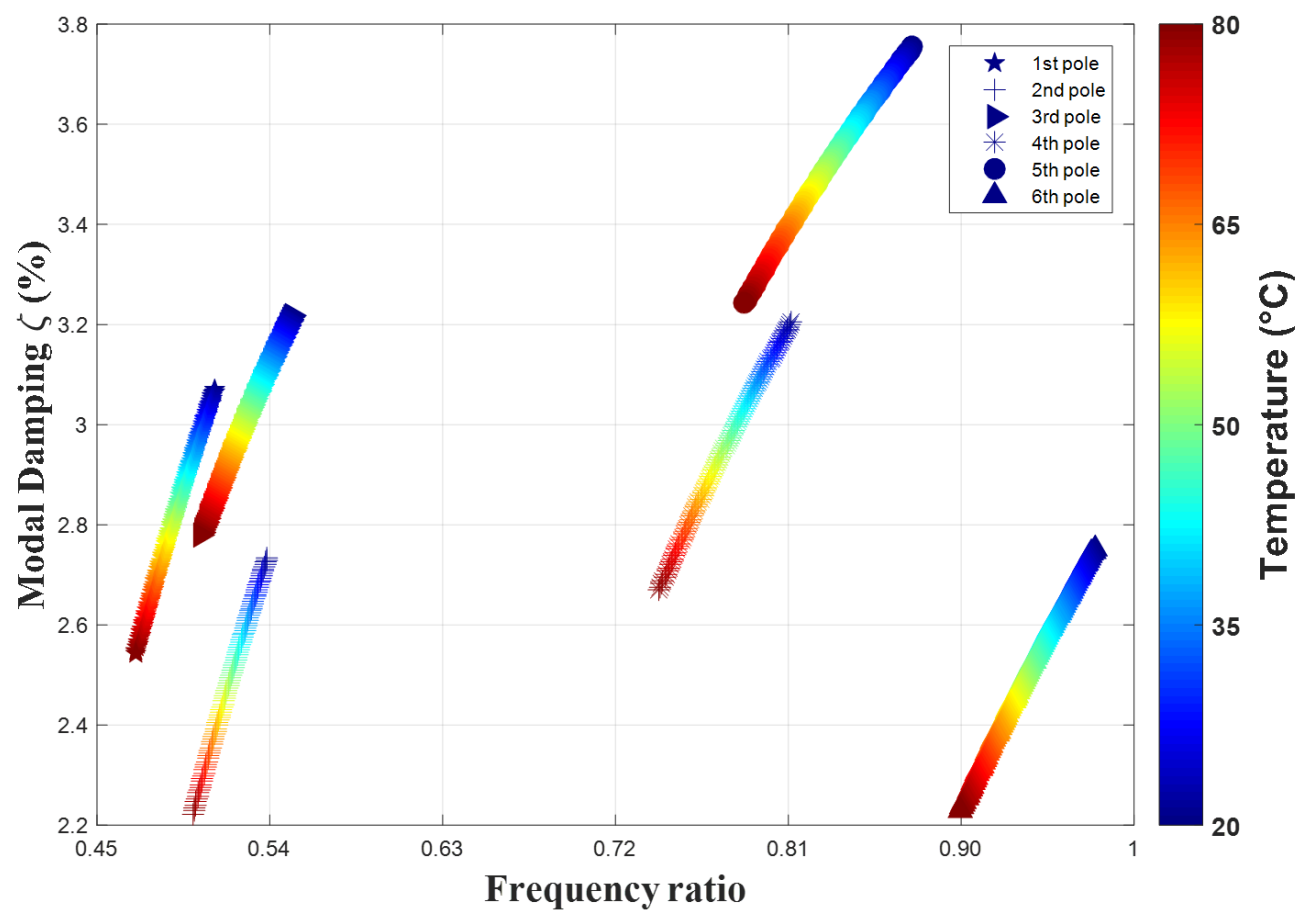

Fig. 7: Evolution of the eigenfrequency and the modal damping for the damper with the silicone rubber part according to the temperature

evolution of the $4^{\text {th }}$ pole (Figure $9 \mathrm{~b}$ ) in this area matches with a mode rotation. The Figure 10 shows this effect more in details. Before the interval (T0), the $4^{\text {th }}$ mode is well identified, then, in the vicinity of $R_{f}=0.6(T c)$, one can observe that a combination of two modes is necessary to describe it. After this interval ( $T 1)$, the rotation is completed and the $4^{\text {th }}$ mode is again well known.

The tools presented in this section allow to take into account the temperature dependency of the elastomers in their rheological model and thus in the finite element model of the damper. For the stronger variations in the poles locations, an index has been used to follow the pole all along the temperature range in order to carry out some comparisons between the damper with the silicone rubber parts and the one with the tBA-PEGDMA parts. The silicone rubber and the tBA-PEGDMA exhibit two different behaviors according to the frequency and the temperature for the domain under study. Theses differences directly affect the behavior of the damper depending on the chosen elastomer. The damper with the silicone rubber parts display an expected and smooth poles variations but the maximum modal damping for the $4^{\text {th }}$ mode is around $3.25 \%$. Contrary to this kind of evolution, the damper with the tBA-PEGDMA parts exhibits a stronger poles variations due to the elastomer phase shifting. However this phenomenon allows the $4^{\text {th }}$ mode of the damper to reach a maximum modal damping of $55 \%$ around $50^{\circ} \mathrm{C}$. 

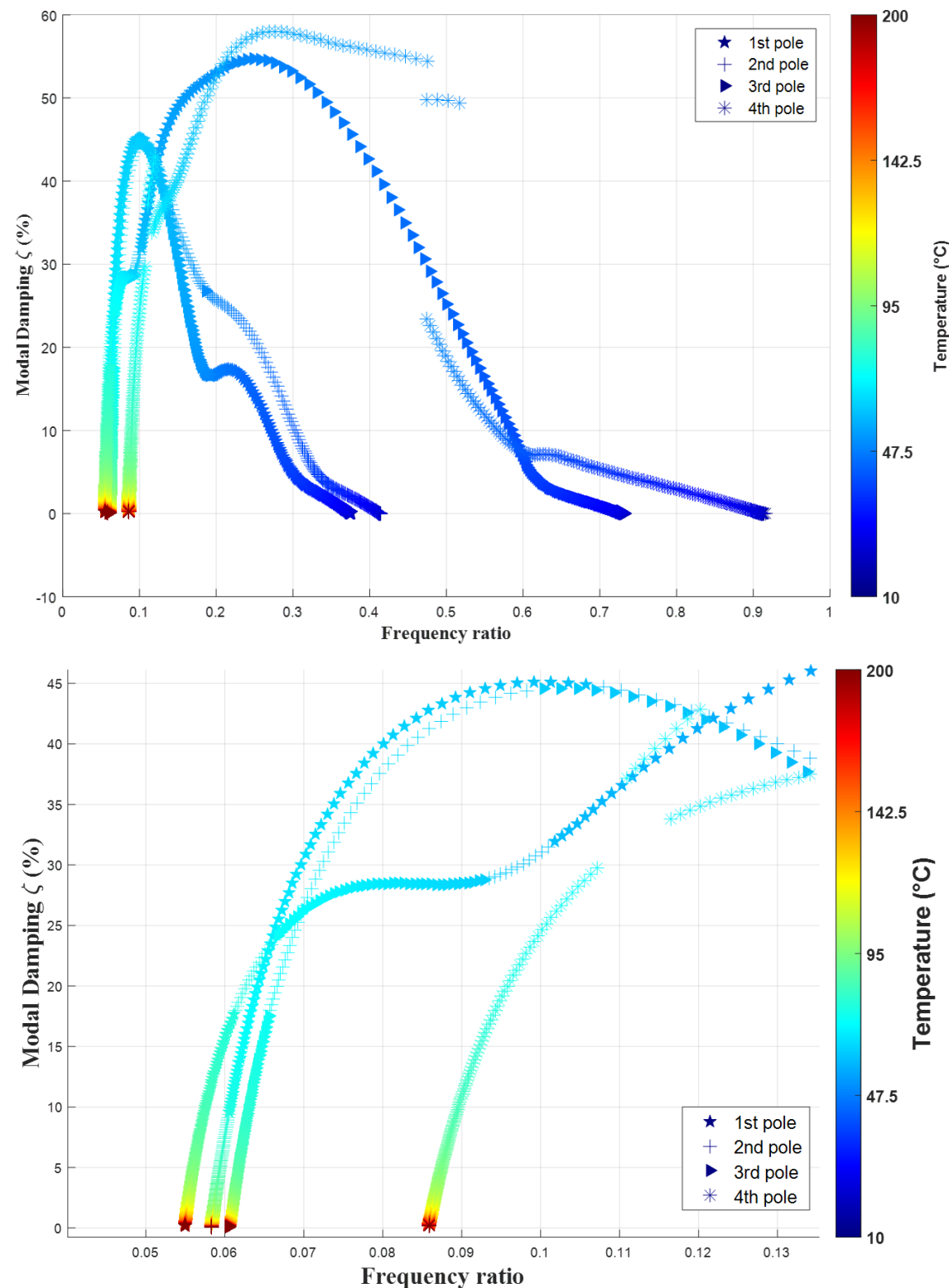

Figure 8: Evolution of the eigenfrequency and the modal damping for the damper with the tBA-PEGDMA part according to the temperature (global view and zoom at high temperature) 


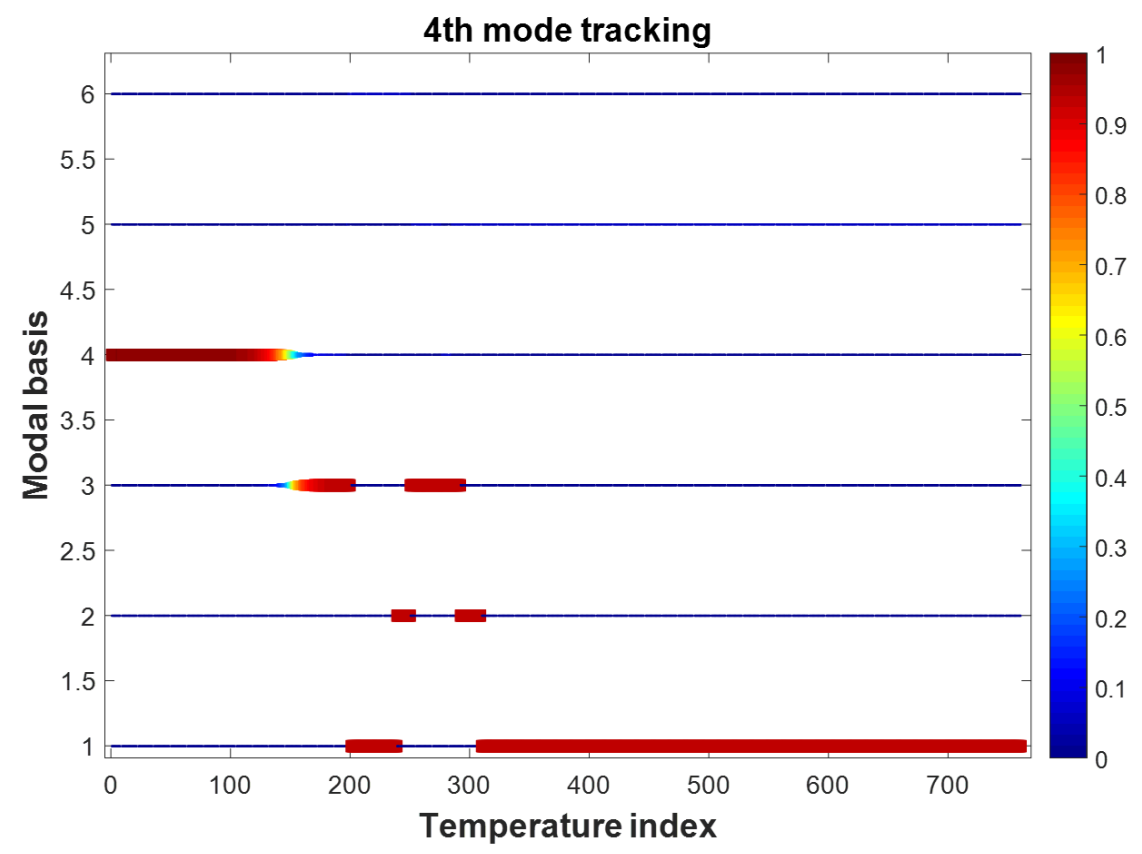

(a) Tracking index for the $4^{\text {th }}$ mode of the damper according to the temperature

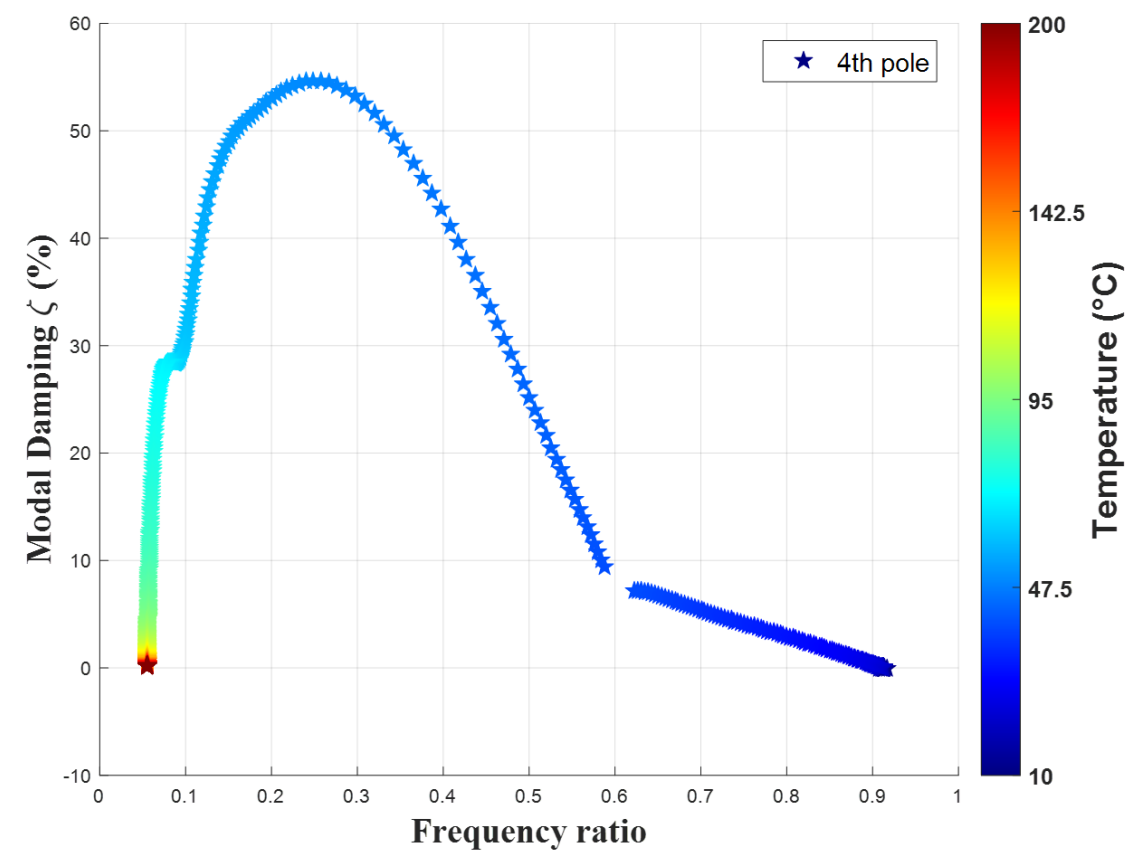

(b) Evolution of the tracked pole associated to the $4^{\text {th }}$ mode according to the temperature

Figure 9: Pole tracking through the temperature range 

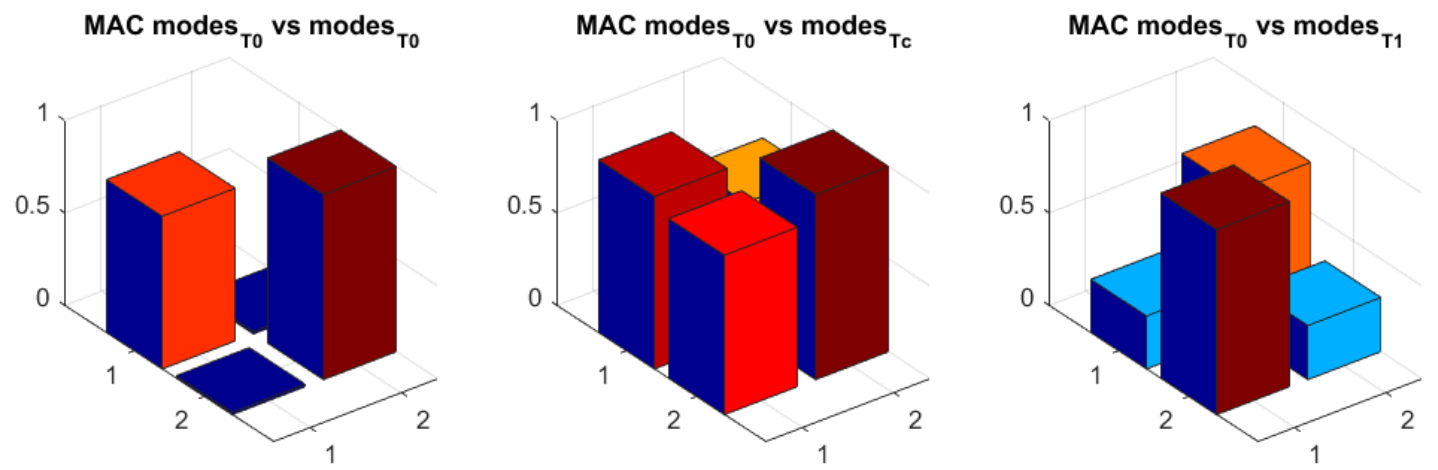

Fig. 10: Zoom on the rotation of the $4^{\text {th }}$ mode around 0.6 


\section{Robust decision making for viscoelastic time and frequency-dependent dampers}

\subsection{Introduction}

Previous sections have shown that the behavior of viscoelastic materials vary with temperature and frequency, this dependency can be introduced using different rheological models in numerical simulations, and it is thus possible to evaluate the damping efficiency of the structures for each material behavior. Simulations are performed for one set of data parameters, and it appears that results can change significantly as parameters vary. When considering lackof-knowledge on input parameters or on the environmental conditions in which structures are used, making decisions on the best design configuration seems unsafe without taking unforeseen uncertainties into account. For instance, the mechanical device presented in this paper is subjected to large derivation on temperature that can consist in sudden variations between $-20^{\circ} \mathrm{C}$ and $80^{\circ} \mathrm{C}$. Due to the position of the damper in the final system, it is hard to accurately know the surrounding temperature of the viscoelastic parts. The effective temperature in which materials are subjected in the real applications may vary, and the profile of variation is an unknown considered as a lack-of-knowledge. In such a thermal environment it would thus be interesting to be able to guarantee a certain damping level, and maybe select a viscoelastic material taking into account both its efficiency and its sensitivity to uncertainties. This question has been adressed in [22, 23] considering probabilistic formulations, such a level of information is not always available and the problem at stake here is for cases where statistical data repartition are unadapted or unknown. Robustness study is the process of evaluating to what extent a design choice is sensible to lack-of-knowledge, and quantifying the performance of this design in an uncertain context.

\subsection{Info-gap decision theory}

The methodology applied in this paper is based on the info-gap decision theory which is well-suited for applications where no probabilistic information is available [24, 25, 26, 27]. It relies on the introduction of three data:

- a numerical model $\mathcal{M}$ to evaluate the output responses of interest $y$ for given input parameters $u$

$$
y=\mathcal{M}(u)
$$

- a performance criterion,

- an info-gap uncertainty model that describes how the input parameters $u$ vary with respect to their nominal values.

In the proposed study the response of interest is chosen to be the modal damping of the $4^{\text {th }}$ mode, and the system model is the finite element model described in section 2 that allows to compute this damping, given all the input parameters (dimensions, materials, …). Equation (Eq. 22) is used for that purpose, based on the eigenfrequencies computed using the Complex Eigenvalue Analysis (CEA) respectively for the silicone rubber and for the tBA-PEGDMA damper. The info-gap model for uncertainty on temperature $T$ is formulated under the following form:

$$
U(h, \tilde{T})=\{T:|T-\tilde{T}| \leq h\}, h \geq 0
$$

where $h$ is the horizon of uncertainty, varying in the study between 0 and $30^{\circ} C$, and $\tilde{T}$ is the nominal temperature fixed at the tBA-PEGDMA glass transition value $\left(50^{\circ} \mathrm{C}\right)$. Hence, the interval covered by the temperature is between $20^{\circ} \mathrm{C}$ and $80^{\circ} \mathrm{C}$. The performance criterion is to ensure that the modal damping of the considered mode $\zeta_{4}$ is smaller than a critical chosen value $\zeta_{c}$ regarding the uncertainty on temperature. Hence, the performance requirement can be expressed as an inequality:

$$
\zeta_{4}(T) \geq \zeta_{c}
$$

The robustness is thus defined as the largest horizon of uncertainty $h$ ( $\max$ ) that can be tolerated on the temperature 
while fulfilling the performance requirement in the worst-case scenario $(\mathrm{min})$ :

$$
\hat{h}\left(\zeta_{c}\right)=\max \left\{h:\left[\min _{T \in U(h, \tilde{T})} \zeta_{4}(T)\right] \geq \zeta_{c}\right\}, \zeta_{c} \geq 0
$$

\subsection{Application}

The damping performances are evaluated for increasing levels of uncertainty. Figure 11 presents the results of the info-gap analysis for the damping of the fourth mode both for the damper with the silicone rubber parts (green stars) and for the one with the tBA-PEGDMA parts (blue stars).

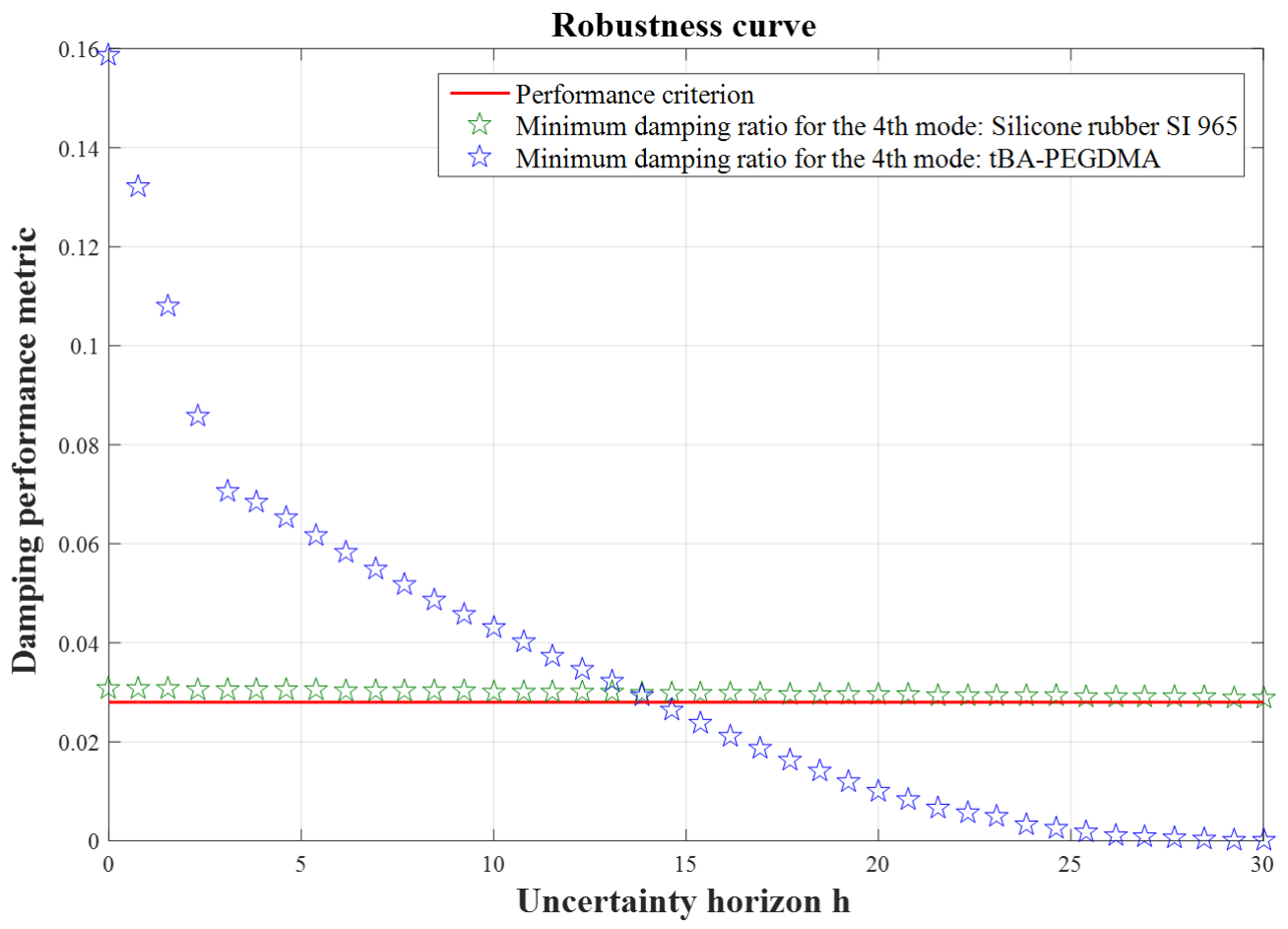

Fig. 11: Robustness curves : Comparison between the minimum modal damping provided by the silicone rubber (green) and the tBA-PEGDMA (blue) according to the uncertainty horizon $h$

Robustness represents the maximum lack-of-knowledge that can be allowed on the nominal value while ensuring that the performance criterion is respected. Curves obtained for the two materials cross together meaning that a change in the both optimal and robust material occur when the uncertainty horizon changes. Let's consider the case of a critical performance value arbitrary chosen at $3 \%$ as plotted in red on Figure 11, the aim is to ensure that this performance is guaranteed even in a uncertain environment. It appears that the damper with the silicone rubber parts provides a modal damping greater than the performance requirement for the entire horizon of uncertainty, while the ensured modal damping is greater than the performance requirement for the damper with the tBA-PEGDMA parts for an horizon of uncertainty smaller than $14^{\circ} \mathrm{C}$. In other words, for this second case, the dynamic model is assumed to be robust for a temperature variation of $\pm 14^{\circ} \mathrm{C}$ around the nominal temperature. Out of this range, the robustness decreases with the growth of the horizon of uncertainty. Different conclusions can be adressed from these results. Considering the case of the tBA-PEGDMA which is a viscoelastic material that exhibits an important variation according to the temperature field, the efficiency can be seriously affected in presence of lack-of-knowledge, and a 
given performance criterion can not be ensured for high level of uncertainty. This phenomena does not occur with the silicone rubber more stable with temperature, and so globally more robust. On the counterpart, this is at the expense of efficiency as the modal damping obtained with the silicone rubber is globally small, and this material could quickly not be a good candidate if the critical performance value is increased. It appears that in such a context it would really be interesting to improve the knowledge on the level of uncertainty, with experimental characterization campaigns for instance, as it could result in changing the viscoelastic material and obtain much better damping performances.

\section{Conclusions}

This paper investigates the best design choice in terms of viscoelastic materials, in presence of lack-of-knowledge on temperature for instance. These materials are likely to present very good damping performances, but their behavior can vary significantly with frequency or temperature. Thus, in an unknown environment, the obtained performances can be very different from expected if the impact of uncertainties is not taken into account. A methodology is presented to study the impact of lack-on-knowledge for temperature, and applied to the case of a damper made with a steel frame and including viscoelastic elements. For that purpose, an original complex eigenvalue analysis is performed on the finite element model of the damper, introducing adequate rheological models for two different viscoelastic materials. The temperature dependency is added to the models using tendencies given by experimental considerations. Two viscoelastic materials with different damping efficiency and temperature influence are considered to evaluate their respective interest. Finally, the damping performances obtained on one chosen eigenmode, and the robustness of the designs are computed using the info-gap decision theory which is well-suited for applications where no probabilistic information is available. The obtained results show that for the same application, the best design choice can change depending on a criteria performance, and on the degree of lack-of-knowledge. The proposed strategy offers a way to quantify both this efficiency and the robustness and help designers to adequatly choose their material for the best performance.

\section{Acknowledgements}

Authors are grateful to the french company THALES LAS Fr for their grateful financial support. This work has been performed in the Framework of EUR EIPHI (ANR-17-EURE-0002).

\section{References}

[1] E. Balmes, S. Germes, Tools for viscoelastic damping treatment design. application to an automotive floor panel, in: International Seminar on Modal Analysis, Leuven, Belgium, 2002.

[2] H. Festjens, G. Chevallier, F. Renaud, J.-L. Dion, R. Lemaire, Effectiveness of multilayer viscoelastic insulators to prevent occurrences of brake squeal: A numerical study, Applied Acoustics 73 (11) (2012) 1121-1128.

[3] M. D. Rao, Recent applications of viscoelastic damping for noise control in automobiles and commercial airplanes, Journal of Sound and Vibration 262 (3) (2003) 457 - 474.

[4] J. Madeira, A. Araújo, C. M. Soares, C. M. Soares, Multiobjective optimization for vibration reduction in composite plate structures using constrained layer damping, Computers \& Structures.

[5] P. Butaud, E. Foltête, M. Ouisse, Sandwich structures with tunable damping properties: On the use of Shape Memory Polymer as viscoelastic core, Composite Structures 153 (2016) $401-408$.

[6] G. Song, L. Li, M. Singla, Y.-L. Mo, Pounding tune mass damper with viscoelastic material, uS Patent 9,500,247 (Nov. 22 2016).

[7] C. Hammami, E. Balmes, M. Guskov, Numerical design and test on an assembled structure of a bolted joint with viscoelastic damping, Mechanical Systems and Signal Processing 70 (2016) 714-724

[8] G. A. Lesieutre, K. Govindswamy, Finite element modeling of frequency dependent and temperature-dependent dynamic behavior of viscoelastic materials in simple shear, International Journal of Solids and Structures 33 (3) (1996) 419-432.

[9] G. Chevallier, F. Renaud, J.-L. Dion, S. Thouviot, Complex eigenvalue analysis for structures with viscoelastic behavior, in: ASME 2011 International Design Engineering Technical Conferences and Computers and Information in Engineering Conference, American Society of Mechanical Engineers, 2011, pp. 561-569.

[10] W. Flügge, Viscoelastic Models, in: Viscoelasticity, Springer Berlin Heidelberg, Berlin, Heidelberg, 1975 , pp. 4-33.

[11] J. Ferry, Viscoelastic Properties of Polymers, Wiley, 1980.

[12] S. Marques, G. Creus, Computational Viscoelasticity, SpringerBriefs in Applied Sciences and Technology, Springer Berlin Heidelberg, 2012.

[13] F. Renaud, J.-L. Dion, G. Chevallier, I. Tawfiq, R. Lemaire, A new identification method of viscoelastic behavior: Application to the generalized maxwell model, Mechanical Systems and Signal Processing 25 (3) (2011) 991-1010. 
[14] P. Butaud, M. Ouisse, V. Placet, F. Renaud, T. Travaillot, A. Maynadier, G. Chevallier, F. Amiot, P. Delobelle, E. Foltête, et al., Identification of the viscoelastic properties of the tba/pegdma polymer from multi-loading modes conducted over a wide frequency-temperature scale range, Polymer Testing.

[15] E. Balmes, Model reduction for systems with frequency dependent damping properties, Office national d'études et de recherche aérospatial. ONERA-Publications.

[16] A.-S. Plouin, E. Balmès, Steel/viscoelastic/steel sandwich shells computational methods and experimental validations, in: International Modal Analysis Conference, Vol. 4062, 2000, pp. 384-390.

[17] L. Sperling, J. Fay, Factors which affect the glass transition and damping capability of polymers, Polymers for Advanced Technologies 2 (1) (1991) 49-56.

[18] T.-L. Teng, N.-K. Hu, Analysis of damping characteristics for viscoelastic laminated beams, Computer methods in applied mechanics and engineering 190 (29) (2001) 3881-3892.

[19] M. Pathan, S. Patsias, J. Rongong, V. Tagarielli, Measurements and predictions of the viscoelastic properties of a composite lamina and their sensitivity to temperature and frequency, Composites Science and Technology 149 (2017) 207-219.

[20] S. V. Joseph, S. Mohanty, Temperature effects on buckling and vibration characteristics of sandwich plate with viscoelastic core and functionally graded material constraining layer, Journal of Sandwich Structures \& Materials (2017) 1099636217722309.

[21] Y. Sefrani, J.-M. Berthelot, Temperature effect on the damping properties of unidirectional glass fibre composites, Composites Part B: Engineering 37 (4) (2006) 346-355.

[22] M. Guedri, A. Lima, N. Bouhaddi, D. Rade, Robust design of viscoelastic structures based on stochastic finite element models, Mechanical Systems and Signal Processing 24 (1) (2010) 59-77.

[23] R. Capillon, C. Desceliers, C. Soize, Uncertainty quantification in computational linear structural dynamics for viscoelastic composite structures, Computer Methods in Applied Mechanics and Engineering 305 (2016) 154-172.

[24] Y. Ben-Haim, Info-Gap Decision Theory, Second Edition: Decisions Under Severe Uncertainty 2nd Edition, Academic Press; 2 edition, 2006.

[25] Y. Ben-Haim, F. M. Hemez, Robustness, fidelity and prediction-looseness of models, Proceedings of the Royal Society of London A: Mathematical, Physical and Engineering Sciences 468 (2137) (2011) 227-244.

[26] A. Kuczkowiak, S. Cogan, M. Ouisse, E. Foltête, M. Corus, Robust expansion of experimental mode shapes under epistemic uncertainties, in: Model Validation and Uncertainty Quantification, Volume 3, Springer International Publishing, 2014, pp. 419-427.

[27] S. Atamturktur, Z. Liu, S. Cogan, H. Juang, Calibration of imprecise and inaccurate numerical models considering fidelity and robustness: a multi-objective optimization-based approach, Structural and Multidisciplinary Optimization 51 (3) (2015) 659-671. 